\title{
In Vitro-In Vivo Correlation for Gontrolled-Release Dosage Forms
}

Russell J. Rackley, Ph.D.

Ciba-Geigy Corporation, Summit, N7

\section{Introdurtion}

In vitro-in vivo correlation (IVIVC) has been defined by the United States Pharmacopeia (USP) Subcommittee on Biopharmaceutics as: "the establishment of a relationship between a biological property produced by a dosage form, and a physicochemical characteristic of the same dosage form" (1). A Food and Drug Administration (FDA) interpretation of IVIVC has been cited as (2): "To show a relationship between two parameters. Typically a relationship is sought between in vitro dissolution rate and 'in vivo' input rate. This initial relationship may be expanded to critical formulation parameters and 'in vivo' input rate." As suggested by either of the cited definitions of IVIVC, physicochemical properties of a dosage form other than dissolution should not be overlooked as an in vitro measurement. However, with respect to quality control testing, more weight tends to be placed on the cumulative dissolution of a dosage form over time as an in vitro indicator of in vivo performance. In vivo performance may typically be assessed in man by rate and extent of absorption of an oral dosage form. For controlledrelease dosage forms, it is especially desirable to determine the cumulative absorption-time profile since this tends to be unique to the formulation. The ultimate goal of an IVIVC should be to establish a meaningful relationship between in vitro behavior of a dosage form and in vivo performance of the same dosage form, which would allow in vitro data to be used as a surrogate for in vivo performance.

Skelly and Shiu (3) have inferred that dissolution testing evolved as a tool for biopharmaceutical investigation of IVIVC throughout the 1950's and 1960's. However, the United States Pharmacopeial Convention has bad the greatest influence on the standardization and general acceptance of dissolution as a quality control tool (4). While there has been a great deal of excitement regarding the utilization of IVIVC in support of scale-up and post-approval changes (5), the current focus appears to be on establishing a biopharmaceutical classification of drugs in support of immediate-release formulations (6). However, immediate practical application of these concepts appears to be with controlled-release formulations. This article is intended to present the reader with an up-to-date overview to approach IVIVC for controlled-release formulations.

\section{Rationale of IVIVE}

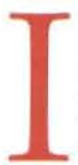

$\mathrm{t}$ is generally easier to establish higher levels of IVIVC with controlledrelease dosage forms than with immediate-release dosage forms. With controlled-release formulations, dissolution (or release from the formulation) tends to be the rate-limiting step in the absorption process. Because different controlledrelease products generally employ different controlledrelease mechanisms, it must be emphasized that IVIVCs for controlled-release formulations must be considered to be product-specific (7). Each formulation development effort for a new drug must be treated on a case-by-case basis.

IVIVC for controlled-release dosage forms will be of benefit to a company when regulatory authorities permit it to be utilized in one or more of the following ways:

- surrogate to bioequivalency studies which might typically be required with scale-up or minor post-approval changes (SUPAC);

- validate the use of dissolution testing and specifications as a quality control tool for process control; dissolution specifications in quality control ranges may be shown to be relevant to in vivo data;

- identify appropriate dissolution conditions for a formulation which result in data relevant to in vivo performance;

- predict in vivo performance of a formulation based on in vitro dissolution data, which may aid in the design of formulation release-time profiles resulting in optimal plasma concentration-time profiles.

\section{Levels of In Vitro-In Vivo Eorrelation}

With respect to the FDA definition of IVIVC, dissolution data provide in vitro data for a dosage form and plasma drug concentration-time data are deconvoluted (see following section) to provide an estimate of absorption data for the same dosage form. The following degrees of correlation of in vitro with in vivo data have been established and accepted in the literature for controlledrelease oral dosage forms $(1,4,8,9)$.

\section{Level A}

Currently the highest level of IVIVC, correlation at this 
level should represent a oneto-one (or point-to-point) relationship between in vitro dissolution and in vivo absorption of a drug from a dosage form. An adequate number of dissolution data points are required to 'profile' in vitro performance. In vivo data must be obtained, such that drug disposition may be defined, in order to use an appropriate deconvolution method to determine the absorptiontime profile of the controlled-release dosage form. The dissolution(in vitro) and absorption- (in vivo) time profiles of the dosage form should ideally be superimposable. The advantage of this level of correlation is that every time point in the dissolution- and absorptiontime profiles is used and there is agreement of the curve shapes of these profiles. A correlation at this level should be considered product-specific.

\section{Level B}

This level of correlation is generally achieved using statistical moment methods. A correlation at this level may be demonstrated by a comparison of mean in vitro dissolution time to mean in vivo dissolution time. In this case, every time point associated with the in vitro data and the in vivo data is taken into account; however, profiles associated with the dissolution and absorption processes may not necessarily reflect each other. A correlation at this level should be considered product-specific, but may also be used for batch-to-batch comparisons.

\section{Level C}

This level of correlation is associated with comparison of a single data point or parameter of in vitro and in vivo data for a drug dosage form. An example could be a comparison of the time required for $80 \%$ dissolution
(T $80 \%)$ of a dosage form and a single pharmacokinetic parameter such as AUC, Cmax, or Tmax. In this case, all of the data points are not taken into account and the profiles associated with dissolution and absorption are not compared. A correlation at this level requires investigation of multiple formulations to demonstrate a changing trend in dissolution and/or pharmacokinetic parameters utilized. This has been a common approach associated with early efforts of IVIVC which were generally applied to immediaterelease oral dosage forms. Ideally, a quantitative relationship (ex. linear) should be expressed at this level; however, a rank-order relationship alone might be considered a low Level C correlation and would be of a qualitative nature.

\section{Mapping}

It has been suggested that "mapping" be used as an alternative to seeking an IVIVC (5). In this case critical formulation variables must be identified and the range allowed for these variables in normal manufacturing must be established. Formulations are made at the limits of the ranges of the critical formulation variables and dissolution is assessed to set dissolution specifications. Formulations used to set limits 


\section{In Vitro-In Vivo Borrelation ... Eont.}

of the dissolution specifications are then subjected to bioequivalency testing, which would justify proposed dissolution ranges. This method appears to represent process validation, rather than demonstration of a correlation.

\section{Deconvolution and Convolution}

Scheme I summarizes the relationships of deconvolution or convolution to IVIVC. Based on a knowledge of the pharma-

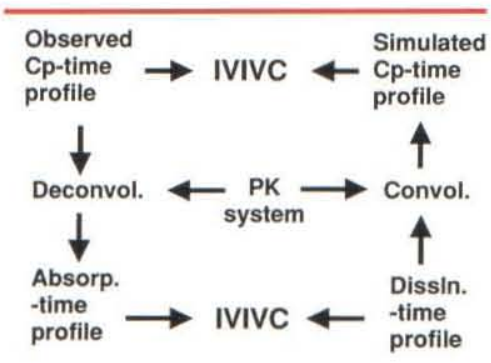

Scheme I cokinetic system for a drug, the plasma concentration-time profile resulting from administration of an oral dosage form may be taken apart, or deconvoluted, to give an absorption-time profile for the oral dosage form in vivo. Also, based on the assumption that release of drug from the controlled-release

formulation is the rate-limiting factor in the absorption process, the absorption-time profile resulting from deconvolution may be considered to be indicative of in vivo dissolution. In general, the use of deconvolution methods, such as Wagner-Nelson (10), LooRiegelman (11), and numerical deconvolution (12-13) requires a fairly good understanding of a drug's pharmacokinetics and pharmacokinetic principles. Some degree of pharmacokinetic modeling for a drug may be necessary to use some deconvo- lution techniques correctly.

Alternately, a knowledge of the pharmacokinetics of a drug may be combined with, or convoluted with, the dissolutiontime profile of an oral dosage form to simulate a plasma concentration-time profile for administration of the controlled-release dosage form to man (Scheme I). Leeson et al. (14) used this process as a method to evaluate prototype controlled-release formulations in vitro without performing more costly and time-consuming in vivo testing, and the concept has been termed 'biorelevant dissolution.' It is essential that sensitivity of the oral controlled-release dosage form be investigated by testing over a diverse range of in vitro testing conditions in order to simulate the in vivo environment (see Dissolution Specifications). As with deconvolution, convolution may only be used when pharmacokinetics for the drug have been established. Simulations resulting from the combination of dissolution data and the pharmacokinetic system are only relative to the in vivo behavior of the rapidly releasing form of the drug, if this is the basis of the pharmacokinetic system (14). A potential stumbling block of these approaches may be related to assumptions regarding absolute bioavailability, as this data may be limited.

\section{Artificial Neural Networks}

In the last ten years, applications of artificial neural networks has grown exponentially.
Training artificial neural networks to a series of in vitro - in vivo correlation data sets is beginning to find some utility in relating dissolution to absorption (or plasma concentration-time profiles) of drugs from controlled release formulations. This methodology has potential advantages over limitations associated with convolution - deconvolution approaches. For more on application of artificial neural networks, interested readers should try to attend introductory short courses, such as that recently taught by R. Erb and A. S. Hussain at the 1995 AAPS Annual Meeting.

\section{Practical Approach to the Level A In Vitro-In Vivo Correlation}

The Level A correlation is currently the highest level of IVIVC recognized and may be specified as a requirement when addressing SUPAC issues without bioequivalency data. The following approaches and examples are meant to demonstrate a starting point for investigation of a Level A correlation.

\section{Dualitative:}

The first test of a Level A correlation is to visualize the dissolution and absorption profiles together to assess the degree of superimposition. If the profiles do not appear to superimpose each other with respect to rate and extent of drug release, then pursuit of a more quantitative correlation may not be warranted. If, however, the profiles appear to 
superimpose each other in this manner, possibly with a lagtime for absorption, then a more quantitative correlation may be explored. For a range of formulations with different release profiles, a rank-order relationship should be observed. Visualization of the dissolution and absorption profiles in Figure 1 demonstrates the correlation at a qualitative level.

\section{Duantitative:}

Linear relationship of absorption as a function of dissolution

Using $x-y$ data pairs representing estimates of dissolution and absorption at common time points, linear regression is performed. By far, this appears to be one of the more popular methods that many researchers use initially to investigate a quantitative relationship between dissolution and absorption data. Superimposable data will have a one-to-one relation and a linear relationship with a slope of one, an intercept of zero, and a coefficient of determination (r-squared) of one. It has been suggested that a $y$-intercept less than zero might be explained by a lag-time in absorption, whereas a significant positive $y$-intercept would not make sense (2). Ideally, the dissolution and absorption data used with this approach should be derived from an adequate number of data points such that each process is fully profiled. Linear regression of the dissolution and absorption data in Figure 1 at common time points was performed.

Hwang et al. (15) have mathematically demonstrated that the lag of absorption-time data in relation to dissolution-time data would be eliminated if in

\section{Qualitative - visualization}

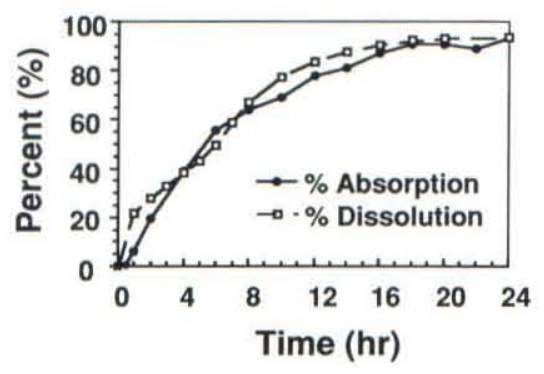

Quantitative - linear relation

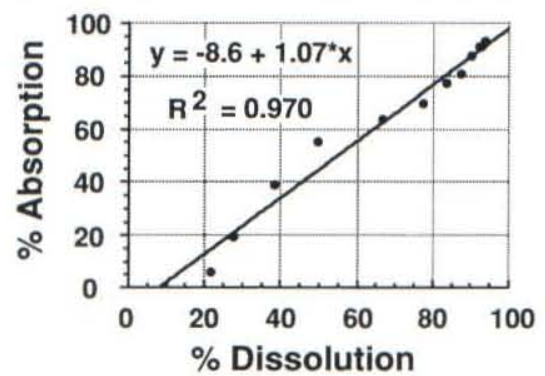

\section{Quantitative - nonlinear relation}

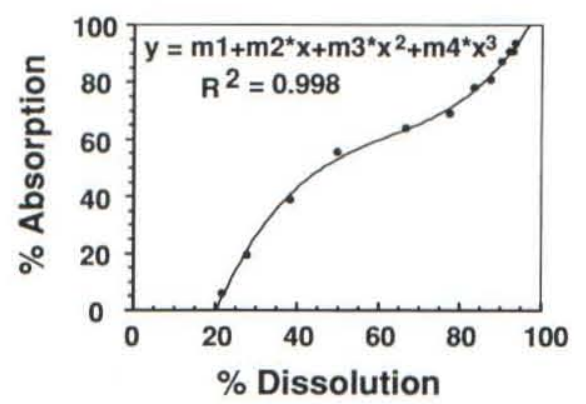

Quantitative - Rescigno Index

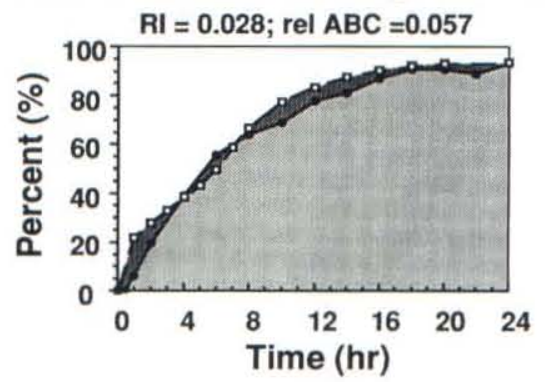

Figure 1

vivo dissolution-time data were estimated using numerical deconvolution methods (12, 13). In cases where drug-release is constant (zero-order), then a simple time-shift in the absorption-time profile, equivalent to the reciprocal of the first-order absorption rate constant, may be used to establish a higher quality Level A correlation (15). When drug release is a slow first-order process, the same approximation appears to apply. The negative $y$-intercept for the linear regression in Figure 1 is indicative of an overall trend for a lag of absorption relative to dissolution data.

Non-linear relationship of absorption as a function of dissolution

There are a variety of possibilities in which non-linear functions may be investigated and the utility of this approach appears to be somewhat empirical. For example, with a nonlin- ear relationship, there may be no parameter targets, analogous to that of linear relationships. And, it is somewhat common to observe some degree of curvature in a plot of absorption versus dissolution at common time points. Some examples of functions that might be used in this case include polynomial equations, Emax (16) and Weibull (17) type equations, and exponential or Gompertz equations (18). The same dissolution and absorption data from Figure 1 was evaluated using a thirdorder polynomial equation.

\section{Rescigno Index}

It has been suggested by Dr. J. Powers (18) that an index reported by Rescigno (19) be considered for comparing the similarity of dissolution- and absorption- time curves. A variation of the Rescigno Index is to relate the difference of area between the cumulative 


\section{In Vitro-In Vivo Gorrelation ... cont.}

dissolution and absorption curves to the area under the cumulative dissolution curve, since dissolution is assumed the independent variable. When the index is calculated in this way, it directly gives the fractional difference of absorption to dissolution. Cumulative absorption and dissolution tend to plateau at some asymptote; therefore, the time up to which absorption and dissolution data are collected should be specified. The dissolution and absorption data in Figure 1, up to 24 $\mathrm{hr}$, was calculated to have a Rescigno Index (RI) of 0.028 using an exponent of one and no weighting. Relative area between the curves (rel ABC) for the same data was calculated as 0.057 , which means that area between the curves was less than $6 \%$ of the area under the dissolution-time curve up to $24 \mathrm{hr}$.

Very little statistical information is available for use of dissolution data as an indicator of bioequivalence ( $\mathrm{T}$. Lin, ref. 18). And thus, there appears to be a need for more statistically based guidelines in the establishment of equivalency of dissolution and absorption profiles. Future investigations of IVIVC and biorelevant dissolution may include the use of nonlinear mixed effect modeling in the evaluation of dissolution and absorption data, for solid oral formulations.

\section{Setting Dissolution Specifications}

With controlled-release formulations, there is an inherent need to 'profile' the release over time. Dissolution specifications define the acceptable range of dissolution-time data and should be representative of the profile and variability associated with a controlled-release dosage form. The USP (20) offers a guide suggesting the time over which the dissolution profile is defined relative to the labeled dosing interval. There should be at least three dissolution time points for dissolution testing of controlled-release dosage forms $(8,18)$ : the first time point should assess dose dumping, the second or more time points should 'profile' the dissolution-time curve, and the last time point should provide information as to recovery of drug in the dosage form. An investigation of the dependence of the formulation on $\mathrm{pH}$ and surfactants is recommended in media of various compositions (8), taking physiological considerations into account. Also, a dependence on dissolution equipment, and range of equipment settings, should be considered in the investigation. Controlled-release formulations that are sensitive to changes in the dissolution environment should be examined to determine in vitro conditions which achieve an optimal IVIVC (5). In this instance the dissolution scientist may play a major role in the optimization of an IVIVC.

Consensus indicates that the historically-based average dissolution data, plus or minus some measure of variation, has been generally used to set dissolution specifications (21).
Experience has demonstrated that the average \pm up to 3.0 SD's may be used as a guide to setting dissolution specifications for some modified release formulations. A more meaningful method of developing dissolution specifications could be through the use of a Level A IVIVC.

Two ways have been suggested to go about this $(5,8)$ : Deconvolution

Limits of variation observed in the actual plasma concentrations in clinical studies are assessed to determine absorption-time profiles (as indicated in Scheme I, page 14). And, the USP has suggested that the $95 \%$ confidence intervals of observed plasma concentrations be deconvoluted to provide absorption-time profiles that may be used as a guide to setting dissolution specifications (20). However, this approach will lead to overinflated specifications for dissolution since variance in the pharmacokinetics associated with the drug may be carried over in the estimation of absorption by the deconvolution process.

Convolution

Dissolution limits set by conventional means may be used to simulate drug input to a pharmacokinetic model (Scheme $I$, page 14). The resulting simulations of plasma concentrations are compared to variation observed in the clinical studies. Here again, caution must be exercised unless provision is made to take into account the variation associated with the pharmacokinetics of 
the drug in the convolution process.

\section{Validation of IVIVG supporting dissolution sperifieations}

Currently, validation of an IVIVC appears to be moving toward the ability to demonstrate that formulations manufactured at the limits of dissolution specifications must individually be shown to be bioequivalent to a formulation representing the mid-point of the dissolution specification. The concept of a Level A correlation would apply and it would probably be expected that there is some control over the ability to manufacture formulations with release rates corresponding to the range of dissolution specifications.

\section{Concluding remarks}

Applicability of IVIVC to SUPAC situations has currently evolved to include demonstration of a relationship of dissolution release specifications, based on variation in dissolution, to variation in plasma concentrations resulting from administration of the same controlled-release formulation. Ideally, an IVIVC should try to demonstrate a relationship between critical formulation parameters and dosage form performance, in vitro and in vivo. The prospect of using an IVIVC correlation as a surrogate for a bioequivalency study appears to be a worthwhile endeavor, as it would have the potential to save time and money. However, investigation and use of IVIVC should be viewed as a long-term investment in the business of drug development.

\section{Acknowledgements}

The author would like to acknowledge Dr. Lewis J. Leeson for training and supervision and the Ciba-Geigy Corporation for experience in this area of research.

\section{References}

1)Pharmacopeial Forum; JulyAugust, 1988; pg. 4160.

2) Cardot JM and Beyssac E, Eur 7 Drug Metab Pharmacokin, 18(1):113-120, 1993.

3) Skelly JP and Shiu GF; Eur 7 Drug Metab Pharmacokin, 18(1):121-129, 1993.

4) Cohen JL, Hubert BB, Leeson LJ, et al.; Pharm Res, 7:983-987, 1990.

5) AAPS/USP/FDA Workshop on Scale-Up of ExtendedRelease Dosage Forms; Crystal Gateway Mariott Hotel, Arlington, VA; Sept. 8-10, 1992; and Skelly JP, Van Buskirk GA, Arbit HM, et al.; Pharm Res 10(12):1800-1805, 1993.

6) Amidon GL, Lennernas H, Shah VP, et al.; Pharm Res, 12:413-420, 1995.

7) Leeson's 1st commandment; DIA meeting, Rockville, MD, Sept. 19-20, 1994.

8) Pharmacopeial Forum, 19(3):53665379; May-June, 1993.

9) Skelly, J. P.; Amidon, G. L.; Barr, W. H.; et al.; 7 Pbarm Sci, 7(9):849-854, 1990; and 7 Controlled Release, 14:95-106, 1990.

10) Wagner JG and Nelson E; 7 Pharm Sci, 52(6):610-611, 1963.

11) Loo JCK and Riegelman $S ; 7$ Pharm Sci, 57:918-928, 1968.

12) Gillespie WR; PCDCON, v.1.1.

13) Chan K, Langenbucher F, and Gibaldi M; 7 Pharm Sci, 76(6):446-450, 1987.

14) Leeson LJ, Adair D, Clevenger J, et al.; 7 Pharmacokin Biopharm, 13(5):493$514,1985$.

15) Hwang SS, Bayne W, Theeuwes F; 7 Pharm Sci, 82(11):1145-1150, 1993.

16) Holford NHG and Sheiner LB; Clin Pharmacokin, 6:429-453, 1981.
17) Langenbucher F; Pharm Ind, 38(5):472-477, 1976.

18) Generic Drugs Advisory Committee, Open Session; Rockville, MD; Jan. 11-12, 1994.

19) Rescigno A; Pharm Res, 9(7):925928, 1992.

20) USP 23; Chapters $\langle 711\rangle,<724\rangle$, and $<1088>$.

21) AAPS/USP Workshop on Dissolution Calibration and Testing; Hyatt Regency Crystal City, Arlington, VA; Sept. 28-29, 1995. 